\title{
EED Inhibitor MAK683
}

National Cancer Institute

\section{Source}

National Cancer Institute. EED Inhibitor MAK683. NCI Thesaurus. Code C133819.

An inhibitor of embryonic ectoderm development protein (EED) and allosteric inhibitor of polycomb repressive complex 2 (PRC2), with potential antineoplastic activity. Upon administration, MAK683 selectively binds to the domain of EED that interacts with trimethylated lysine 27 on histone 3 (H3K27me3), which leads to a conformational change in the EED H3K27me3-binding pocket and prevents the interaction of EED with the histone methyltransferase enhancer zeste homolog 2 (EZH2). Disruption of the EEDEZH2 protein-protein interaction (PPI) results in a loss of H3K27me3-stimulated PRC2 activity and prevents H3K27 trimethylation. This decrease in histone methylation alters gene expression patterns associated with cancer pathways and results in decreased tumor cell proliferation in EZH2-mutated and PRC2-dependent cancer cells. PRC2, a histone $\mathrm{H} 3$ lysine 27 methyltransferase and multi-protein complex comprised of EZH2, EED and suppressor of zeste 12 (SUZ12), plays a key role in gene regulation, especially during embryonic development. EZH2, the catalytic subunit of PRC2, is overexpressed or mutated in a variety of cancer cells. EED is essential for the histone methyltransferase activity of PRC2 because EED directly binds to H3K27me3. 